\title{
Phase-locked arrays of unstable resonator semiconductor lasers
}

\author{
J. Salzman ${ }^{\text {a) }}$ and A. Yariv \\ California Institute of Technology, Pasadena, California 91125
}

(Received 24 February 1986; accepted for publication 30 June 1986)

\begin{abstract}
A phase-locked array of several unstable resonator semiconductor lasers is demonstrated. Single lateral mode was obtained for currents $I<2.5 I_{\text {th }}$, and partial spatial coherence for $I \leqslant 5 I_{\text {th }}$, with an optical output power of $0.95 \mathrm{~W}$.
\end{abstract}

Laser cavities of the unstable resonator (UR) type have the potential for increasing the mode volume of a laser oscillator while maintaining high discrimination between transverse modes. ' This property makes UR geometries very attractive for semiconductor lasers since it allows an increase in the lateral dimension of the laser (and an increase in the emitting area) while maintaining single lateral mode operation and eliminating filamentation. UR semiconductor lasers have been demonstrated previously, ${ }^{2-5}$ and single lateral mode operation with output powers up to $400 \mathrm{~mW}$ was observed. ${ }^{5}$ By extrapolating these results to the case of an optimal cavity design for high power operation ${ }^{6}$ we estimated that these devices have the capability to emit $1-2 \mathrm{~W}$ of optical output.

However, a further increase in the output power of a single UR semiconductor laser in the convex mirror configuration is difficult to achieve due to the intrinsic limitations in the geometrical parameters of the cavity: in order to avoid filamentation, the curvature radius $R$ of the etched mirrors has to be smaller than a certain critical value, ${ }^{2}$ and for a fixed value of $R$, the cavity losses increase with the length $L$. The width of the UR laser is also limited by total internal reflection at the output plane. Therefore, limiting values of the mode volume and the emitting width of UR semiconductor lasers are $\sim 1.5 \times 10^{4} \mu \mathrm{m}^{3}$ and $-100 \mu \mathrm{m}$, respectively.

One way to overcome these limitations is to couple laterally several UR semiconductor lasers. Phase locking by lateral coupling is easily achieved in this case because the oscillating field in each laser spreads, thus favoring mutual injection. An additional property of the UR laser array is that the aberrations in the optical field produced by local inhomogeneities in the lasing medium are reduced in the multicavity structure. In fact, the aberrations of the total output field are just the average of the field aberrations in each of the individual cavities. ${ }^{7}$

In this work, the operation of phase-locked arrays of several UR semiconductor lasers is demonstrated. On a GaAs/GaAlAs double heterostructure grown by liquid phase epitaxy, a laser mirror was etched in the form of several circular arcs as shown in Fig. 1 (a). The curvature radius of the circular arcs is $R=15 \mu \mathrm{m}$, with $10 \mu \mathrm{m}$ center separation. The etching of the mirror facets, and the rest of the fabrication steps are identical to those of the previously reported semiconductor lasers with nonplanar mirrors. ${ }^{2,5} \mathrm{~A}$ scanning electron microscope photograph of the etched facet is shown in Fig. 1(b). The width of the combined struc-

\footnotetext{
" Present address: Bell Communication Research, 331 Newman Springs Road, Red Band, NJ 07701-7020.
}

ture is determined by the upper ( $p$-side) metallization, varying from 80 to $120 \mu \mathrm{m}$ in the presently reported devices. As shown in Fig. 1(c), the half-symmetric configuration was used. The planar mirror was formed by cleaving, thus allowing to fabricate devices of different lengths ( 100 $\mu \mathrm{m} \leqslant L \leqslant 500 \mu \mathrm{m})$. The lasers were tested under pulsed conditions with $200 \mathrm{~ns}$ pulses, and $1 \mathrm{kHz}$ repetition rate.

A distinguishing characteristic of symmetric of halfsymmetric UR lasers is the presence of a virtual source behind the output facet. ${ }^{1,4}$ In our case, an array of virtual sources is expected. In Fig. 2, the intensity distribution at the planar mirror (near field) and at two different planes behind the mirror is recorded, for a $210-\mu \mathrm{m}-1$ ong and $120-\mu \mathrm{m}$-wide device. The near field $(z=0)$ resembles that of a single UR laser. ${ }^{5}$ At $z=-40 \mu \mathrm{m}$, the ripples in the intensity distribution start exhibiting a well defined array pattern and this pattern sharpens with increasing distance and develops into a series of very well defined virtual point sources at $z=-65$ $\mu \mathrm{m}$. The expected depth $z$ of these virtual point sources is given approximately by $z_{p}=\left(R L+L^{2}\right)^{1 / 2} / n$, where $n$ is the effective index of refraction of the laser medium. In our case $z_{p}=-63 \mu \mathrm{m}$ in good agreement with the measured value. It is worth mentioning that shorter lasers show even sharper virtual sources, but they do not exhibit a well defined Gaussian-like envelope. This may be due to a higher influence of nonuniformities in gain, in the shorter devices.

For eventual applications of the UR array, it is useful also to observe the near-field intensity distribution at the etched facet. Since every circular arc at this facet extends beyond the critical angle for total internal reflection, the output is in the form of spots, as shown in Fig. 3. Note that there are more than 12 spots even when the injected region width is $120 \mu \mathrm{m}$, because of optical pumping in the regions adjacent to the laser by the spreading field. The appearance of a double array of spots in the photograph of Fig. 3 is due to a Lloyd's mirror ${ }^{8}$ formed at the bottom of the etched region.

The most salient feature of UR array is its far-field intensity distribution (FF) with peaks at regular angular intervals (Fig. 4). Well defined peaks with deep minima between them are obtained only for certain values of the device length $L$. This is shown in Fig. 5, in which the FF of two lasers of the same wafer with different lengths are given. In Fig. 5(b) part of the light is scattered into secondary peaks with different angular periodicity from that of the main peaks. (Note also that the maxima in Fig. 5 are narrower than these of Fig. 4, because they result from a wider device.) By changing further the length, the FF becomes more complicated. The reproducibility of these observations was confirmed by measuring many devices for each value of L. 


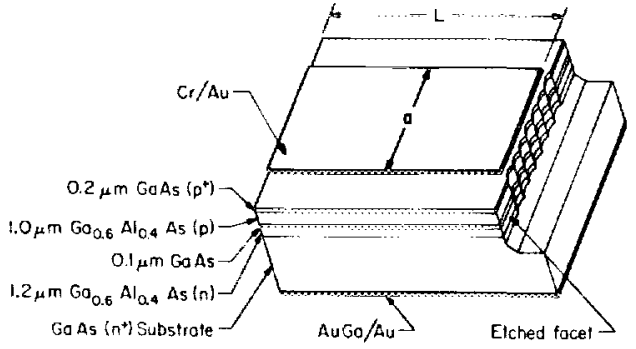

(a)

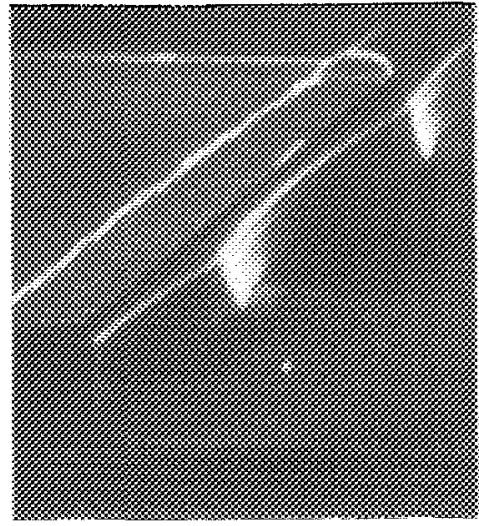

(b)

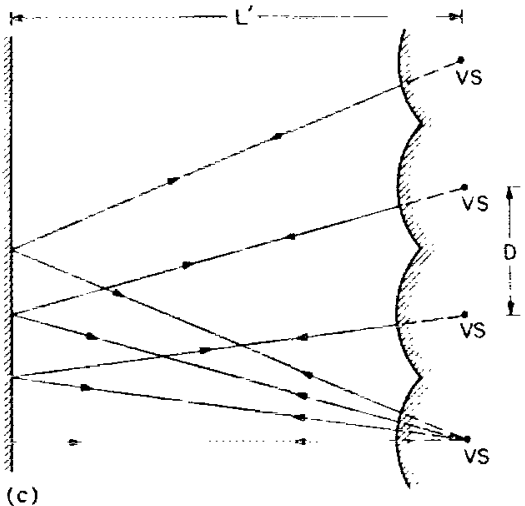

(c)

FIG. I. (a) Schematic drawing of the unstable resonator laser array. (b) Scanning electron microscope photograph of the etched mirror. (c) Composite cavity (not to scale) showing the optical axes in several pairs of interacting elements. VS, virtual sources.

The characteristic FF of the UR array and its dependence on $L$ can be qualitatively explained by the following simple model: we assume that the field close to the optical axis of each UR is not seriously distorted by the coupling to the other cavities. By incorporating lateral periodicity into the (approximate) waveguide solutions of a single UR, ${ }^{9}$ it can be shown that the lowest order lateral mode of the combined structure appears as emanating from the array of virtual sources, with these virtual sources in phase. The maxima in the interference pattern originated by the array of virtual sources are at angle $\theta_{1}$ given by $\tan \theta_{1}=n_{1} \lambda / D$, where $\lambda$ is the wavelength in the laser medium, $D$ is the separation between adjacent sources, and $n_{1}=0, \pm 1, \pm 2 \ldots$ On the other hand, the composite cavity will favor reflection at par-

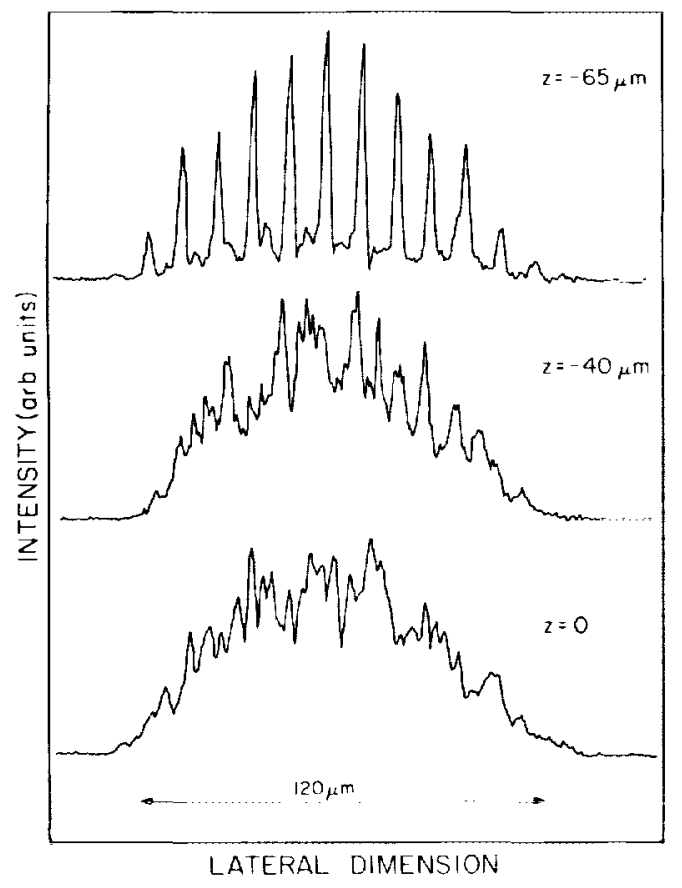

FIG. 2. Near-field intensity distribution (lower trace) and intensity distribution with the imaging system focused at different planes behind the output facet. The label $z$ in each trace is the distance between the focusing plane and the output facet and its accuracy is $\pm 5 \mu \mathrm{m}$. ticular angles $\theta_{2}$ such that the field can nearly reproduce itself after one round trip and a lateral shift of $n_{2} D$. These angles are given by [Fig. 1(c)] $\tan \theta_{2}=n_{2} D / 2 L^{\prime}$, where $L^{\prime}$ is the distance between the virtual sources and the planar mirror, and $n_{2}=0, \pm 1, \pm 2, \ldots$ By requiring that the angles $\theta_{1}$ and $\theta_{2}$ coincide, we obtain the relation $F^{\prime}=2 n_{1} / n_{2}$, with $F^{\prime}=D^{2} / L ' \lambda . F^{\prime}$ is a modified Fresnel number and plays a role similar to $N_{\text {eq }}$, the equivalent Fresnel number in Siegman's analysis of UR's.' (The proof that $F^{\prime}=2 n_{1} / n_{2}$ for $n_{1}$ and $n_{2}$ small integers also minimizes the cavity losses will be given elsewhere. ${ }^{10}$ ) For example, by requiring that the first interference maximum $\left(n_{1}=1\right)$ coincide with the favored angle between next neighbor elements $\left(n_{2}=1\right)$, we obtain $L^{\prime}=225 \mu \mathrm{m}$ which corresponds to $L=210 \mu \mathrm{m}$. This is the device length from which Fig. 2 and Fig. 5(a) were obtained, and very close to that of Fig. $4 .{ }^{11}$

The external quantum efficiency of the UR array, $\eta_{d}$, was measured and compared with that of Fabry-Perot lasers fabricated from the same wafer, $\eta_{d, F p}$. At injection levels lower than twice the threshold current $I_{\mathrm{th}}$, the external quantum efficiency was $\eta_{d} \simeq 0.85 \eta_{d, F p}$. At higher injection

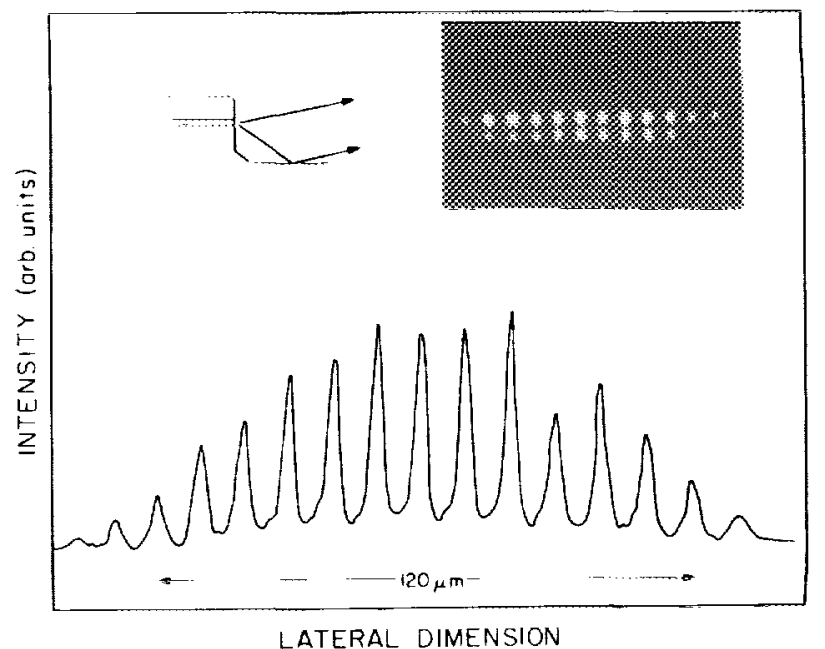

FIG. 3. Near-field intensity distribution at the etched faced of the UR array. The insert shows a photograph of observed field, with a schematic explanation of the double pattern seen. 


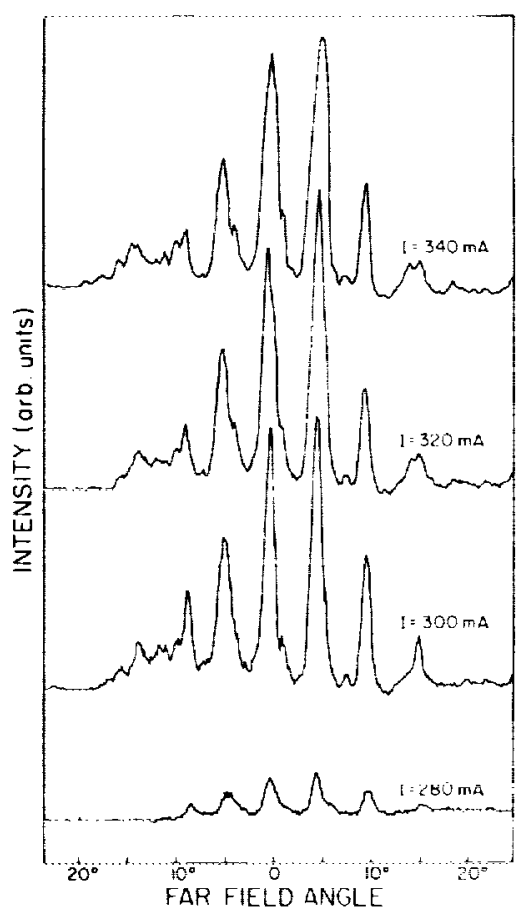

FIG. 4. Far-field intensity distribution for a device width $W=80 \mu \mathrm{m}$ and length $L=205 \mu \mathrm{m}$, for different values of the injection current.

currents, the Fabry-Perot lasers showed nonlinearities and changes in the near-field intensity distribution. Catastrophic failure happened at output powers of $-600 \mathrm{~mW}$. In the UR array lasers, the light versus current characteristic was linear up to a current of $5 I_{\mathrm{th}}$, with an output power of $0.95 \mathrm{~W}$ (the maximum current injected and output power were limited by our current source).

The spatial coherence of the array was investigated by measuring the lateral coherence of these lasers as a function of the injection current, by imaging the laser near field on a variable double slit, and observing the interference pattern produced by the transmitted light. ${ }^{4}$ High visibility of the interference fringes was observed up to $2.5 I_{\mathrm{th}}$. At higher currents, the visibility of the fringes decreased gradually, and at $5 I_{\mathrm{th}}$ the UR array was still partially coherent $(\sim 0.4$ visibility of fringes). That suggests that saturation of the oscillating cavity mode, and the appearance of additional lateral modes is gradual in a way similar to that suggested for phaselocked arrays ${ }^{12}$ fonmed by index or gain-gained waveguides.

The enission characteristics of the UR array suggest that this laser source can be very useful for distributing its output into several ports (multiplexing). This can be done in the near field (Fig. 3) or in the far field (Fig. 4). The basic geometry of this laser is suitable for up-scaling, and this

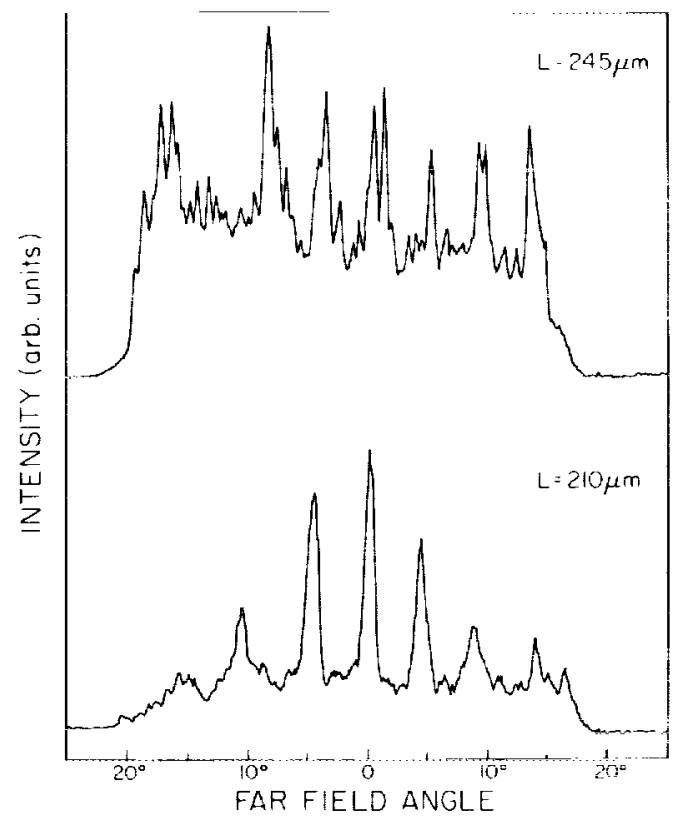

FIG. 5. Far-field intensity distribution at $I=1.2 I_{\mathrm{th}}$. (a) $W=120 \mu \mathrm{m}$, $L=210 \mu \mathrm{m}$; (b) $W=120 \mu \mathrm{m}, L=245 \mu \mathrm{m}$.

property makes the UR array a potential candidate for high power applications.

This research was supported by grants from the Air Force Office of Scientific Research, the Office of Naval Research, and the National Science Foundation. J. Salzman would like to acknowledge the financial support of the Bantrell Postdoctoral Fellowship and the Fullbright Fellowship.

'A. E. Siegman, Proc. IEEE 53, 277 (1965)

'J. Salzman, T. Venkatesan, R. Lang, M. Mittelstein, and A. Yariv, Appl. Phys. Lett. 46, 218 (1985).

${ }^{3}$ R. R. Craig, L. W. Casperson, O. M. Stafsudd, J. J. J. Yang, G. A. Evans, and R. A. Davidheiser, Electron. Lett 21, 62 (1985).

${ }^{4}$ M. Mittelstein, J. Salzman, T. Venkatesan, R. Lang, and A. Yariv, Appl. Phys. Lett. 46, 923 (1985)

5J. Salzman, R. Lang, T. Venkatesan, M. Mittelstein, and A. Yariv, Appl. Phys. Lett, 47, 445 (1985).

'J. Salzman, R. Lang, and A. Yariv, Electron. Lett. 21, 820 (1985).

${ }^{7}$ M. Y. Konopnicki and M. E. Smithers, Appl. Opt. 22, 947 (1983).

${ }^{\mathrm{X}} \mathrm{M}$. Born and E. Wolf, Principles of Optics (Pergamon, New York, 1965) p 262 .

'L. W. Chen and L. B. Felsen, IEEE J. Quantum Electron. QE-9, 1102 (1973).

"J. Salzman, R. Lang, and A. Yariv (unpublished).

"The model presented here may provide only a qualitative description. The use of a geometrical, paraxial approximation, and the assumption that the cleaved mirror is in the far field of the array of virtual sources should be relaxed in a more detailed analysis.

${ }^{12}$ K. L. Chen and S. Wang, Appl. Phys. Lett. 47, 555 (1985) 